\title{
Wearable Devices Components for High Jump Sport: An Overview
}

\author{
Muhammad Faris Roslan ${ }^{1}$, Afandi Ahmad ${ }^{1,2^{*}}$ \\ ${ }^{1}$ Reconfigurable Computing for Analytics Acceleration (ReCAA) Research Laboratory, \\ Microelectronic and Nanotechnology-Shamsuddin Research Centre (MiNT-SRC), \\ Universiti Tun Hussein Onn Malaysia (UTHM), P. O. Box 101, 86400 Batu Pahat, Johor, MALAYSIA \\ ${ }^{2}$ Department of Computer Engineering, Faculty of Electrical and Electronic Engineering, \\ Universiti Tun Hussein Onn Malaysia (UTHM), P. O. Box 101, 86400 Batu Pahat, Johor, MALAYSIA \\ *Corresponding Author \\ DOI: https://doi.org/10.30880/ijie.2020.12.01.003 \\ Received 16 September 2018; Accepted 2 January 2019; Available online 31 January 2020
}

\begin{abstract}
Wearable devices like FitBit, Garmin and Amazfit smartwatch have become popular in recent years. Athlete like swimmers and runners mostly use smartwatch to track speed of the swim or run using embedded global positioning system (GPS) sensor in the wearable devices. Unfortunately, high jump sports still on the old style in tracking the movement of the jump by using vision system. Thus, this paper presents an overview on related work, existing methods and developments on the area of wearable sensor for high jump sports. The analysis of high jump sports related works in terms of main injury and parameters to prevent the injury are being analysed. The used of biosensors in recent wearable devices has been also reviewed in term of the types and issues of sensor that need to implement in the wearable device. Other than that, the processing methods that contains Internet of Thing (IoT) gateway device, network transmission and real-time monitoring system (RtMS) are proposed to develop a complete wearable device. Finally, the main injury parameters of the high jump sports and sensors related to the parameter has been reviewed and an overall idea of processing method RtMS has been summarised.
\end{abstract}

Keywords: High jump sport, real-time, biosensors, monitoring, wearable device, gateway, Internet of Thing (IoT)

\section{Introduction}

Wireless sensor networks (WSNs) have attract a lot of attention from the production and research community during the last decade by the development of the wearable device that help in many sports areas like running, soccer, basketball and tennis [1]. In addition to this development, a variety system prototypes and marketable products have been produced to handle the user demand, which aim to provide real-time data for health condition that gain from the athlete to increase the athlete performance or technique. This data is very important to the coach, to monitor their athletes progress activities and to change the method of training if there stay in low performances in winning sports competitions. There are a lot of wearable sports device in the world [1]. However, there is no wearable device for high jump athletes. Most devices focus on larger sports competitions such as soccer and running.

High jump is one (1) of the track and field sports in which the athlete must jump individually over a horizontal bar placed at measured heights without dislodging it [2]. At the professional level, athletes run towards the bar and use the Fosbury Flop method of jumping, leaping head first with their back to the bar [3]. The simplest technique for clearing the 
bar is the "Scissors Style", in which a straight run-up is used [4]. From there, the evolution of high jump techniques has been improved by the following techniques known as the "Western Roll", the "Straddle" and the "Fosbury Flop", which is the trendiest at present.

The high jump athletic performance and improvement of the record level depend on many variables needed to be considered. Conventionally, most of the high jump athletes used vision system [3], [5]-[17] that is high-speed camera to view their image data. However, these data need to be analysed by the researchers first, before the final result were produced and given to the coaches and athletes. This outdated vision system was not very efficient, and it is costly. Thus, to improve these systems the used of real-time monitoring system (RtMS) is to give real-time result with the advanced wireless communication technology that communicate using WSNs for activity recognition. Currently, RtMS [18]-[34] has been applied to various applications in the world and it is an important practical value for industry production and sports. It enables data administrators to review all the processes and functions performed on the data in real-time, or as it happens, through bar and graphical charts on a main monitor interface.

In this paper, the current state in research and development of wearable for high jump is reviewed by summarising and comparing the attributes of the most capable current achievements of numerous worldwide developments and marketable products. Section 2 presents the related work of the high jump sport. In Section 3, the most significant and commonly engaged biosensor technologies that used in wearable system are explained. Section 4 discusses the processing method, connection and monitoring system of the device. Finally, Section 5 summarises the related work and Section 6 concludes the paper.

\section{Main Monitoring Parameters in High Jump Sport}

High jump is a track and field sport that requires athletes to run and jump by crossing the bar. To maintain and control the jump, athletes need to jump constantly during training, and this can cause injury at the parts of the leg, if they are practicing too much. Many athletes tend to maximise the performances while risking their safety and body condition. Therefore, the discussion about the injury monitoring parameters of the athletes and the stages that caused major injury need to be divided into certain levels of high jump sport. High jump sport comprises three (3) stages where Stage I is the approach run, Stage II is the take-off and the last Stage III is the flight phase or crossing the bar for landing process. Therefore, to improve performance, stages I and II are significant parts to maximise the performance of high jump athletes [3], [5]-[17] while reducing the injury during the run and take-off jump simply by monitoring the main parameters. This section explains the major injuries in this sport and the main parameter that has to be taken into consideration to develop the wearable devices.

\subsection{Injury}

Jumping technique may be enriched through particular strength trainings such as hopping, bounding and box plyometric. These trainings are tense on the body due to the minor contact times and high ground response forces involved. Most of the jumping injuries are caused by the following factors:

(i) High ground reaction forces [35]:

The ground reaction forces are high in jumping activities and will be even higher on simulated surfaces. This will cause anatomical structures to allow the load through high stress trainings when jumping. However, an efficient use of advanced ground reaction forces is useful in a performance aspect as illustrated in Fig. 1. Dangerous amounts of jumping on a hard surface will finally lead to injury. There are high intensity jumping activities performed in high volume to get an athlete to perform at the highest level. These makes the jumper carries the injuries throughout their jobs.

$$
\text { Initial posture Knee onset }
$$

Ankle onset
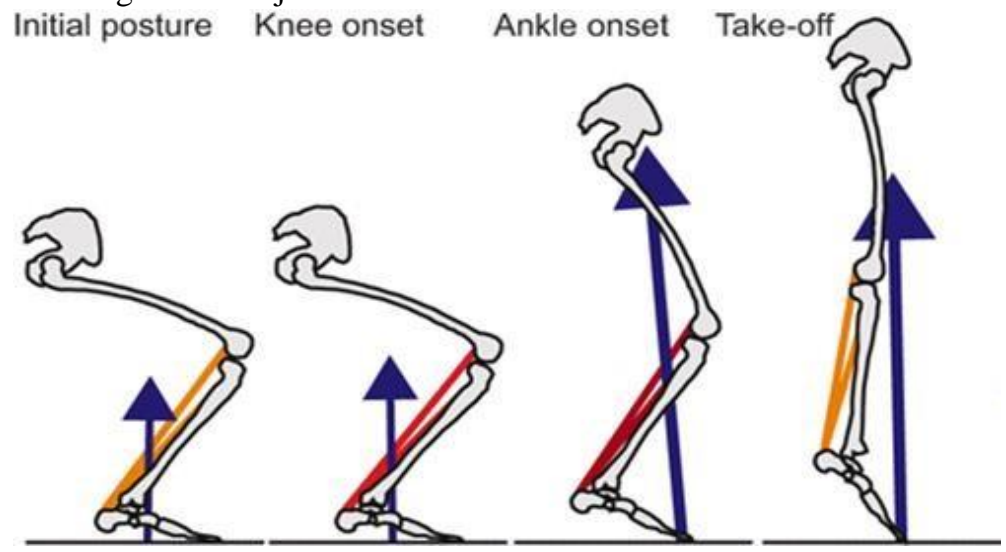

Initial posture

Knee onset

Ankle onset

Take-off

Fig. 1 - The high ground reaction force of the jumper [36] 
(ii) Poor physical preparation [9]:

The poor physical preparation tends to turn the amateur jumpers into increasing the possibility of developing jumping related injuries because their training level is normally too low for regular high intensity jumping. Some jumpers may have a specific anatomical structure, muscle imbalance or inflexibility that will influence them to have an injury. The coaches normally can identify these problems after the athlete gets hurt with quite low training loads.

(iii) Overuse [37]:

Common coaching practice makes professional jumpers to train with high intensity activities in high capacity so that they will perform at maximum height during jumping. This will cause some parts of their body to be overused and damaged as shown in Fig. 2.

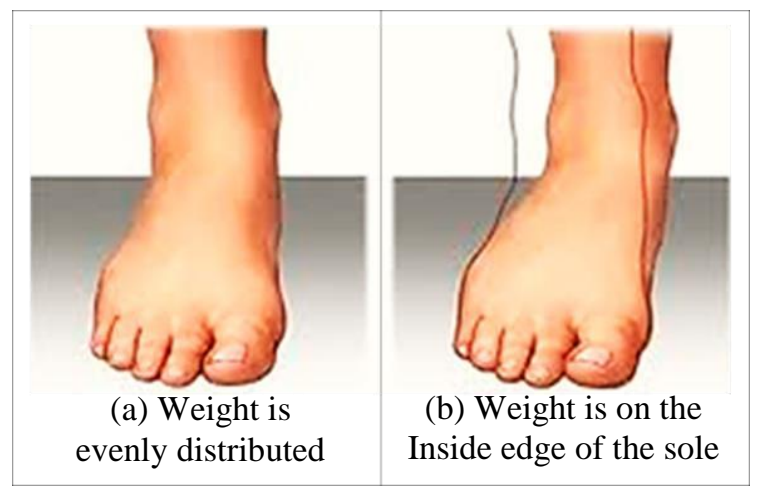

Fig. 2 - (a) Normal and (b) Overuse leg that affect during high intensity training [37].

Langer and Langerova [13] concluded that injuries on the high jumpers' locomotor system were divided into the seven parts of the body which includes foot, shank, knee, femur, pelvic girdle, spine and others. A brief note by [13] and the results are as depicted in Fig. 3 reveals that most injuries of the locomotor system are caused by one or a combination of two (2) or more of the following factors:

(i) high values of reaction process at the take-off;

(ii) inadequate training load;

(iii)imbalanced fitness training;

(iv) imperfect technique of the take-off; and (v) anatomical disposition to injuries.

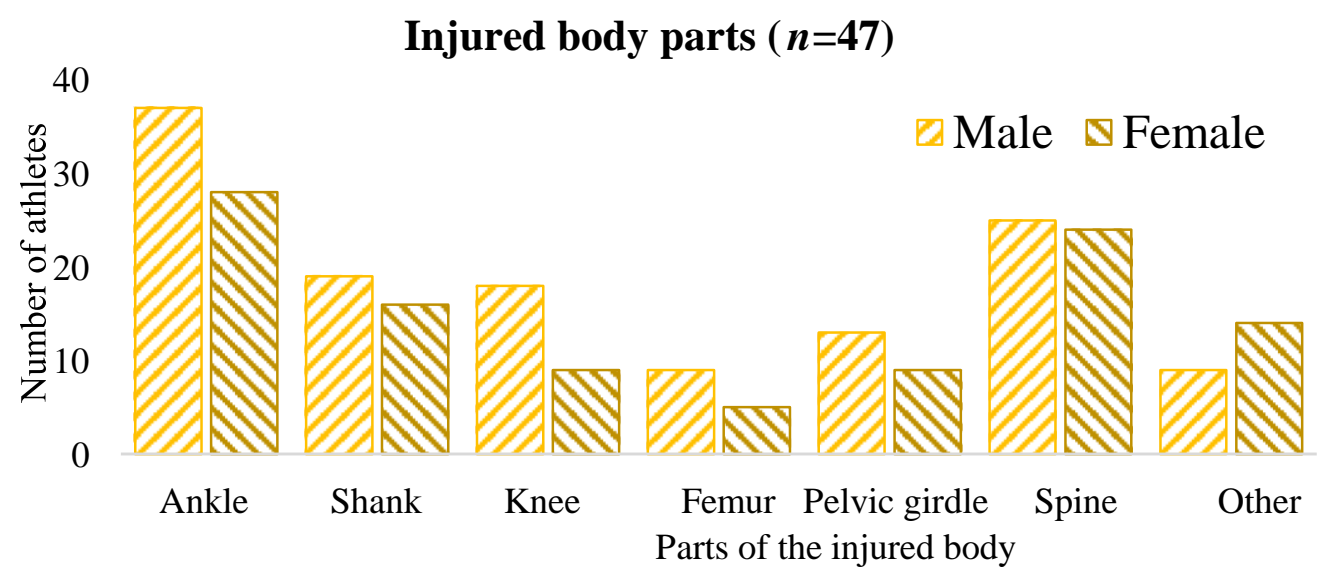

Fig. 3 - The most frequently injured parts of the body with the top male and female high jumpers in the motivated sets from 1983 to 2005 [13]

\subsection{Stage I}

This section discusses the approach level of jump before take-off stage. It is one (1) of the most important stages that distinguish the height results of the jump. High jumpers run at moderate speeds and set down the foot at the take-off position. In 1990, Alexander [10] found that; a moderate run at about $7 \mathrm{~ms}^{-1}$ for normal male jumper can improve the height of jump. However, in the recent year which was in 2016 [8], the best approach speed result has increased to $8.4 \mathrm{~ms}^{-}$ ${ }^{1}$. Meanwhile, other scholars [6], [7], [15]-[17] conducted the experiments and the results obtained were almost the same 
where they are in the range of $5.73 \mathrm{~ms}^{-1}$ to $7.78 \mathrm{~ms}^{-1}$. It can be determined in Table 1 that in between 1990 to 2016 , there are no significant changes in the horizontal approach speed. This is because the moderate low run-up speeds used by high jumper have given benefits to control the take-off and obtain good Fosbury Flop flight during the bar clearance.

Table 1 - Speed parameter of high jump athlete during approach run

\begin{tabular}{cc}
\hline Refs. & Speed $\left(\mathbf{m s}^{-\mathbf{1}}\right)$ \\
\hline$[6]$ & 7.78 \\
{$[7]$} & $6.00-7.50$ \\
{$[8]$} & $7.70-8.40$ \\
{$[10]$} & 7.00 \\
{$[15]$} & 7.40 \\
{$[16]$} & $5.73-7.26$ \\
{$[17]$} & 6.64 \\
\hline
\end{tabular}

\subsection{Stage II}

Take-off stage is the next important stage to improve the maximum height of the jump. A lot of parameters required to be concerned. However, the main parameters that mostly used high-speed camera were recorded during the take-off stage and they are angle (planted leg and knee flexion) and ground reaction forces. Table 2 summarises the outcomes of the previous researches [3], [5], [6], [10]-[12], [14]-[17]. Fig. 4 shows the angle of plant and knee of the high jump leg that have been considered [16]. From the experiments, the angle of plant leg that needs to be gained at the last touchdown is around $30^{\circ}$ to $53^{\circ}$ while the best flexion angle of knee during take-off is at $144^{\circ}$ to $170^{\circ}$. The ground reaction forces were measured in many ways; some of the researcher used force plate sensor to calculate the maximum torque [5] and force [7] around the leg of the jumper. Whereas, others used force platform to measure force [17] and power impulses [8].

Table 2 - Ankle and knee angle parameters of high jump athlete during take-off

\begin{tabular}{ccc}
\hline & \multicolumn{2}{c}{ Parameters $\left(^{\circ}\right)$} \\
\cline { 2 - 3 } Refs. & $\mathbf{1}$ & $\mathbf{2}$ \\
\hline$[3]$ & 30.00 & - \\
{$[5]$} & - & $167.00-170.00$ \\
{$[6]$} & 45.10 & 168.14 \\
{$[10]$} & 45.00 & - \\
{$[11]$} & $32.50-40.80$ & 144.00 \\
{$[12]$} & 30.00 & - \\
{$[14]$} & - & 157.00 \\
{$[15]$} & 53.00 & 157.00 \\
{$[16]$} & 34.00 & - \\
{$[17]$} & - & 177.00 \\
\hline
\end{tabular}

Note:

1: Angle of leg plant, 2: Angle of knee flexion
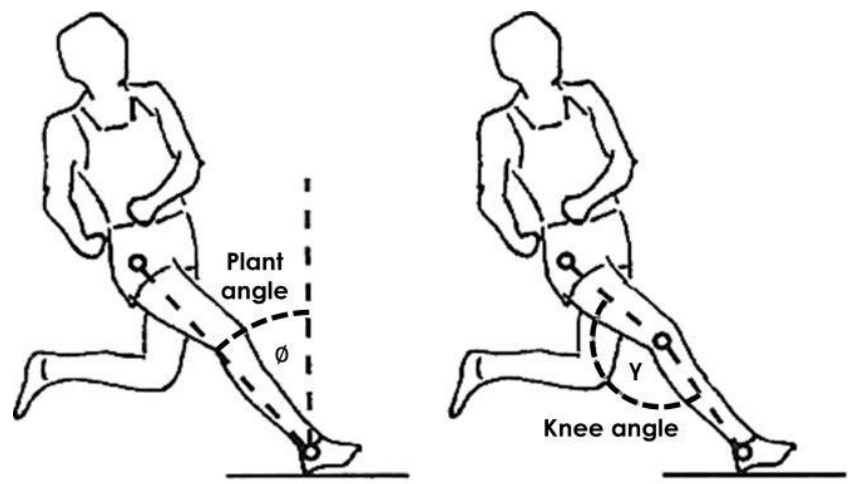

Fig. 4 - Plant and knee angle at take-off phase [16] 


\section{Biosensor Technology}

The massive amounts of biosensors produced by semiconductor industry from all over the world [1] caused the researchers to tend to explore and experiment the sensors through humans. Most of the sensors create human life in safe mode in terms of health situation besides securing their lifestyle. The sensors were placed to humans like a wearable device to study the lifestyle of the human as well as to keep track on their behaviour like heart beat monitoring device [38] which is used to track the value of the heart beat either it is in good or bad conditions and tells the human to stay in healthy life or directly send the data to the professionals like medical doctor or supervisors. This topic describes, about the type of sensors and challenge that need to confront.

\subsection{Type of Sensors}

There are a lot of sensors in the market that are used in variety applications such as sports. In sports, some wearable devices were developed to track and analyse athletic body movement activities such as walking and running where most of the devices track movements using inertial measurement unit (IMU), barometer, global positioning system (GPS), flex, force sensing resistor (FSR) and heartbeat sensor. An overview of the type of sensors is discussed in the following subsection.

\subsubsection{Inertial Measurement Unit (IMU)}

The IMUs are the electronic modules that collect angular and linear velocity with the help of magnetic field to prevent drift [39]. IMUs are normally a standard feature of smartphones, smartwatches and fitness trackers that contains three (3) sensors (accelerometer, gyroscope and magnetometer) and it produces a precise input data position of the object after it is being processed by the microcontroller unit (MCU) or microprocessor unit (MPU). IMU has been regularly used in many sports device like running, soccer, basketball and tennis to learn the athlete motions [1]. The following is a brief description of the IMU sensor:

(i) Accelerometer Sensor

A sensor that measure a non-gravitational acceleration by responding to vibrations. Once an object increases the velocity from zero (0), it will respond the vibrations connected with such movement and converted it to acceleration value. It measures accelerations in each of its axis [33].

(ii) Gyroscope Sensor

It used gravity of earth to determine orientation. It measures the angular velocity with respect to the body axis. It consists of a rotor disk that freely rotates where the rotor is fixed onto a spinning axis in the centre of a greater and more stable wheel. As the sensor turns, the rotor remains static to specify the central gravitational pull [33].

(iii) Magnetometer Sensor

A sensor that is used to measure magnetic field strength is called the magnetometer sensor. It is used to assist and calibrate against orientation drift from accelerometer and gyrometer. This sensor is very important to minimise the error of drift. Flying devices like drone need to use this to encounter the drift that effected from the wind or ship that effected from the wave of the water flow [33].

\subsubsection{Flex Sensor}

Flex sensor is a sensing material that provides angular displacement measurement. It is very slim, and it measures the resistance value of the bending motion when it is applied to a device for sensing fitness equipment. In gaming technology, it is obviously being used in virtual reality (VR) device to enhance the user's experiences. By using flex sensor to detect finger gestures implemented in the glove controller, it can create more control elements inside the virtual environment [40]. Other than that, it can be used in rehabilitation training [41] and when flex sensor is attached to the knee and arm joint, the movements result of the patients can be seen mostly for athletes who experienced bad injury.

\subsubsection{Force Sensing Resistor (FSR) Sensor}

Force sensing resistor (FSR) is a sensor that allows human to detect physical pressure, squeezing and weight. In previous works, FSRs were included in the experiment that as they were used to measure force-myography (FMG) signals to estimate torque by the wrist [42], to measure the pressure at the heel during stepping for foot ulcer screening [43] besides monitoring quadriceps muscle force activity at the gym [44].

\subsubsection{Heartbeat Sensor}

A heartbeat sensor or commonly called pulse sensor used optical power variation where light is absorbed during its path through the blood as the heartbeat changes. The simple heartbeat sensor consists of a light emitting diode (LED) as a transmitter and a receiver of reflection light similar to light detecting resistor (LDR) or a photodiode. The receiver 
output is in a form of electrical signal and is proportional to the heartbeat rate. This sensor is implemented at large for commercial sports watch to check the fitness of the athletes [45].

\subsection{Potential Issues When Attaching Sensors to the Athlete Body}

In order for the sensor system to provide the appropriate setting for the recognition of activity, there are still a number of challenges that must be faced. The raw data produced by the sensor sometimes lagged in many ways. Human behaviour and technical issues of the sensor are mostly the biggest challenges that the researcher needs to solve up until today.

\subsubsection{Sensor Accuracy}

Most of the sensor networks are designed to work over a specified range. With the good accuracy, it is easy for the researcher to process the sensor data. Sometimes, the data have an error that needs to be resolved using mathematical algorithms. Meanwhile, other inaccuracies take place when it exceeds the datasheet range specification and thus causing damage to the sensor or the controller itself.

\subsubsection{Placement of Sensor}

Every human in this world is different in terms of shapes of the body. Thus, the sensor used to record their activity needs to be placed at the correct position to achieve the objective of the experiment. Incorrect position of sensor will minimise the accuracy of the result, although it is a few millimetres from the correct position, and it will require the researcher to repeat the experiment again and again.

\subsubsection{Power Consumption}

There are a lot of sensors that used low-power consumption. However up until now, it is still not enough to manage the total power consumption with the combination of input sensor, processing unit and the actuator output that use too much power. As a result, the lithium-polymer (Li-Po) battery is widely used and it offers slightly higher energy and it is also mostly thinner than the conventional lithium-ion (Li-Ion). Nixon et.al [46] presented the improvement of GPS sensor used by soccer athletes who previously drained the battery life. With this improvement, the device is capable to extend the power consumption of the system. Thus, it is better for the wearable devices that need smaller and lighter battery with better power consumption.

\subsubsection{Usability}

Some devices contain one (1) sensor that can sense one human activity at one task. While the others, can sense a lot of human activities and do a lot of tasks. Wearable fitness smartwatch [47] is one (1) of the examples that can sense heartbeat, calorie burned, percentage of battery used and track the step of the day. This indicates that the usability of the sensors in the devices is important and efficient to the user to keep track their fitness level.

\section{Internet of Things (IoT)-based Solution as a Processing Method}

To build a wearable device that implement IoT-based solution as a processing method, various parameters must be considered such as sensor processing unit (controller), communication between controller and server, and monitoring devices for the users to see the results. Thus, this section discusses about the IoT gateway devices, wireless network and RtMS.

\subsection{Gateways Devices}

There are billions of devices connected to every single part of the world via IoT. To have connectivity in the IoT system, there are devices called IoT gateway. It is the main important device used to collect sensor data and then decode it between sensor protocols and processes sensor data before sending it ahead to the server or cloud.

In human sensory system, senses are used to see, hear, taste, smell and touch. Based on the human sensory system, human created millions of sensors that can measure the heat, light intensity, number of products and many more. The sensors are mainly used only to detect the required inputs. However, IoT system is not just a serial communication or sensing data. It is about two-way communication that is sensing and controlling the data.

The gateway devices comprehend a lot of ways to be connected where in hardware implementation it includes wired (serial ports, Ethernet) and wireless (Wi-Fi, Bluetooth), whereas in software it can be connected via message queue telemetry transport (MQTT) protocols. Both hardware and software can be connected with or without the same control and security setting. To communicate between sensor and server, it needs a controller to process the data.

MQTT is a low bandwidth setting messaging protocol that is designed for machine-to-machine (M2M) telemetry to publish/subscribe the message [48]. MQTT acts as many-to-many communication protocol to communicate messages among many clients over a dominant broker that is the main server. It attains free connection, an important package concerned with the principle of splitting manufacturer and user by permitting clients to publish with the responsibility 
on the broker to choose where to path the messages. These clients interconnect to a middleware which is the server and it also operated as broker to simplify the connection using MQTT connectivity protocol.

The middleware simplifies monitoring of client like wireless sensor nodes over local besides remote network.

In home automation system [49] demonstrated in Fig. 5, the MQTT communicated the clients by a two-way communication using hardware of integrated sensor and actuator via wireless network. With the good, practical, secured and inexpensive sensor, network for converting traditional home into a smart home and also the easiness to connect to the Wi-Fi at almost every home, can transform into something at a low cost and is affordable to provide a wide range of devices to operate at every home using MQTT protocol. Other than that, there is a lot of clouds that connect over the MQTT and it is also simple to connect and free to be used.

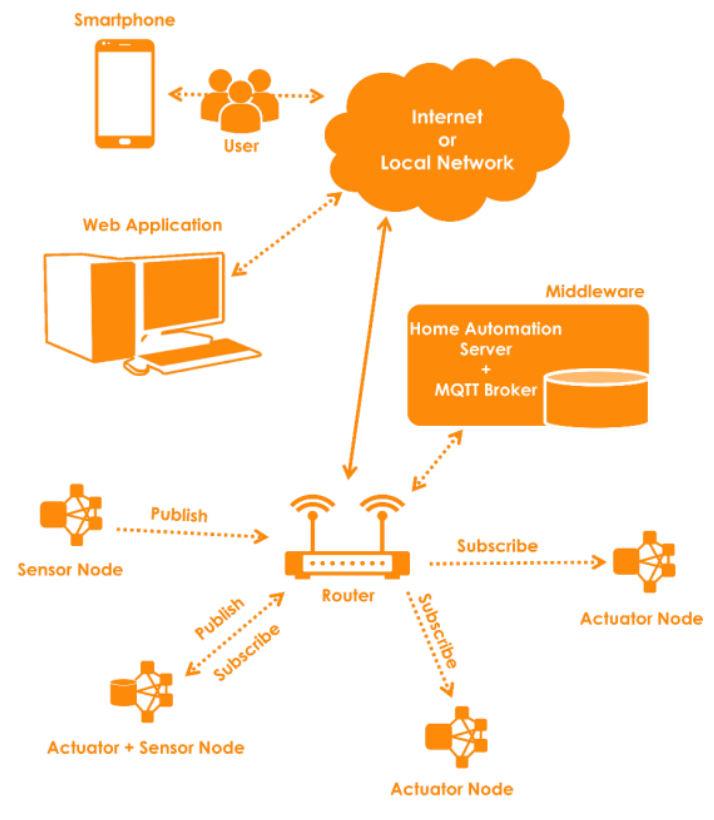

Fig. 5 - Overview of MQTT system using sensor and actuator [49]

Most of the low-performances controller, generate a lot of raw data from the sensor and the data will then be processed by the server after going through the gateway to handle the controller processing speed, such as throughput and latency [48], [50]-[57]. While for high-performance controller, the data are processed before the gateway and it minimises the processing rule in the server. This low-performance controller is used to process and maintain the throughput and latency of the data and most of it has low-power consumption, wireless communication, small in terms of size and low-cost like ESP8266 [58] and Arduino Yun Mini [59] MCUs. It is good for application areas like sports since it is capable to attach, to any part of the body without disturbing the athlete's movements. The data sent through the gateway is only the raw data. Thus, all sensors data can be managed and controlled from the server to the users.

\subsection{Network Transmission}

With the advances of the modern communication technologies, the use of radio-frequency (RF) like Bluetooth and $\mathrm{Wi}$-Fi are greatly used in wireless network. There are a lot of wearable smartwatches in the markets that use Bluetooth to connect and synchronise their data through smartphone apps between cloud storage and the smartwatch. Bluetooth is also more convenient in terms of low-power consumption. Preceding medical electronic research [60], it explains that the heart sound detection used Bluetooth digital stethoscope, thus it helps a lot to monitor the patients suffering from chronic diseases. Nevertheless, the use of Bluetooth in this study is only for connecting the heartbeat sensor to the computer, while, to monitor, the system is at a long distance for medical check-up or professional consultancy, and therefore the computer needs to be connected to Wi-Fi to extend the range.

ZigBee [61] is created based on the personal-area network (PAN) standard (IEEE's 802.15.4). It is usually measured as substituted to $\mathrm{Wi}-\mathrm{Fi}$ and Bluetooth for some applications as well as low-power devices that are incapable for high bandwidth. Besides, it is capable to send data through a big area, where it passes through multiple hops, and all the ZigBee modules are capable to be controlled with low-power by using battery. For this purpose, it is widely used in manufacturing and home automation systems such as switches in the house that control wirelessly.

The ZigBee itself has three (3) types of modules and they include the coordinators, routers, and terminal as illustrated in [62] and in Fig. 6. Coordinators create the link and collect data corresponding to security keys while routers perform as a centre node and communicate data from other modules. Lastly, terminal or end of modules is low-power devices that can connect with coordinators and routers, but it is unable to transmit data to other end devices. 


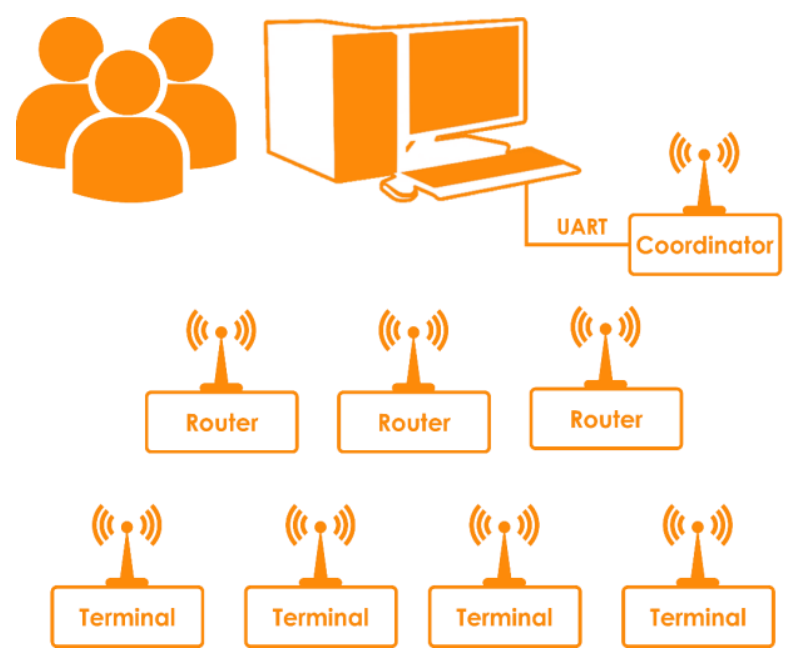

Fig. 6 - Overview of ZigBee communication setting [62]

However, there are some advantages and disadvantages of Wi-Fi and ZigBee in terms of power consumption, throughput and range. ZigBee is suitable for the devices that require a low data transmission, long power consumption, and long distance in radius. In terms of power consumption, Wi-Fi are commonly used in gadget like laptops and smartphones that usually has battery life of one (1) or two (2). Meanwhile, the ZigBee device application has a longer power consumption that is more than a year.

Standard Wi-Fi has a maximum bitrate of $54 \mathrm{Mb} / \mathrm{s}$, whereas ZigBee only allows $250 \mathrm{~kb} / \mathrm{s}$. This is because there is such a big difference concerning the two (2) standards, the throughput and latency of both Wi-Fi and ZigBee need to be considered to apply at the device's application. On the other hand, ZigBee has better access support range that can length until 1,000 metres range, whereas most of the Wi-Fi transmission is only capable to support the range of about 100 metres. Therefore, Wi-Fi is mostly applied at home and ZigBee is widely used to transmit long distance data like in the jungle or sea. Table 3 shows the comparison of Bluetooth, Zigbee and Wi-Fi protocols [63].

Today, Wi-Fi is greatly used in IoTs to connect between clients and server. The presence of Wi-Fi and Internet connection in every home these days is very pleasant. It helps regular house to turn into a viable smart home that contains RtMS, for example, a home security system [64] that used Wi-Fi scanner, heat and door opening sensor in relations to safety of the house in order to distinguish strangers and owner of the house.

Table 3 - Comparison of the Bluetooth, Zigbee and Wi-Fi Protocols [63]

\begin{tabular}{lccc}
\hline Standard & Bluetooth & Zigbee & Wi-Fi \\
\hline IEEE specification & 802.15 .1 & 802.15 .4 & $802.11 \mathrm{a} / \mathrm{b} / \mathrm{g}$ \\
Frequency band & $2.4 \mathrm{GHz}$ & $3.1-10.6 \mathrm{GHz}$ & $2.4 \mathrm{GHz} ; 5 \mathrm{GHz}$ \\
Max signal rate & $1 \mathrm{Mb} / \mathrm{s}$ & $250 \mathrm{~kb} / \mathrm{s}$ & $54 \mathrm{Mb} / \mathrm{s}$ \\
Nominal TX power & $010 \mathrm{dBm}$ & $(-25)-0 \mathrm{dBm}$ & $15-20 \mathrm{dBm}$ \\
Number of RF channels & 79 & $1 / 10 ; 16$ & $14(2.4 \mathrm{GHz})$ \\
Channel bandwidth & $1 \mathrm{MHZ}$ & $0.3 / 0.6 \mathrm{MHz} ; 2 \mathrm{MHz}$ & $22 \mathrm{MHz}$ \\
Max number of cell nodes & 8 & 8 & 2007 \\
\hline
\end{tabular}

Based on the previous studies [60]-[62], [64], even though both Bluetooth, ZigBee and Wi-Fi connections have the ability to connect wirelessly, so far, there are still drawbacks. The benefits of Bluetooth are low-power consumption and minimal set-up cost compared to Wi-Fi connection. Whereas, Wi-Fi and ZigBee connections have the capability to connect more user-client as well as high-speed data transmission rate, quick scanner and coverage signal and long range connectivity [65].

\subsection{Real-time Monitoring System (RtMS)}

These days, all data are collected, analysed and monitored from the sensor and actuator in real-time. Smartphone is one (1) of the RtMS device that in every second gives real-time data and user monitoring experiences. These RtMS devices are mainly controlled by using serial or parallel processing unit and it is depending on the need of the applications. The increasing inputs of the sensors, complexity to process the data and additional algorithms in it have resulted to the requirements of high-speed controller used to process the data and to produce the outputs in real-time.

To solve these issues, most of the controllers are capable to process the sensor input raw data and send the data directly via gateway to the server. Since most of the servers are built to handle high speed input and output, the server is best to process the input sensor data and send it back to the controller to activate any actuator or display the data through 
any mobile devices. This can be seen by some previous IoT studies [27], [30], [33], [66]-[68] as illustrated in Table 4, that had many sensors attached to the subject and the server acts as a main processing unit to control all the data and the built apps will be monitored by the users.

Table 4 shows that Bluetooth and Wi-Fi are widely used in many sports application to monitor the real-time data, this is because, it is easy to connect and most of the users such as athletes and coaches used smartphone or any mobile devices that has Bluetooth and Wi-Fi connection. Meanwhile, ZigBee is rarely used between the end-user as it is widely used in industry to connect multiple devices in a wide range. The operating system (OS) like Android is generally used to run the smartphone brand of Samsung, Sony and HTC, while only one (1) brand runs iPhone OS (iOS) in the device which is Apple. Both OS have shown quite a good performance throughout the year. Therefore, many mobile apps were produced to operate for both OS. This is difficult for the users who have different OS at both wearable device and smartphone to connect or synchronise their data. Some of the users still used the traditional method to monitor their data through webpage only, while others used mobile apps to monitor the data anywhere they are.

Table 4 - Summary of RtMS for sports application

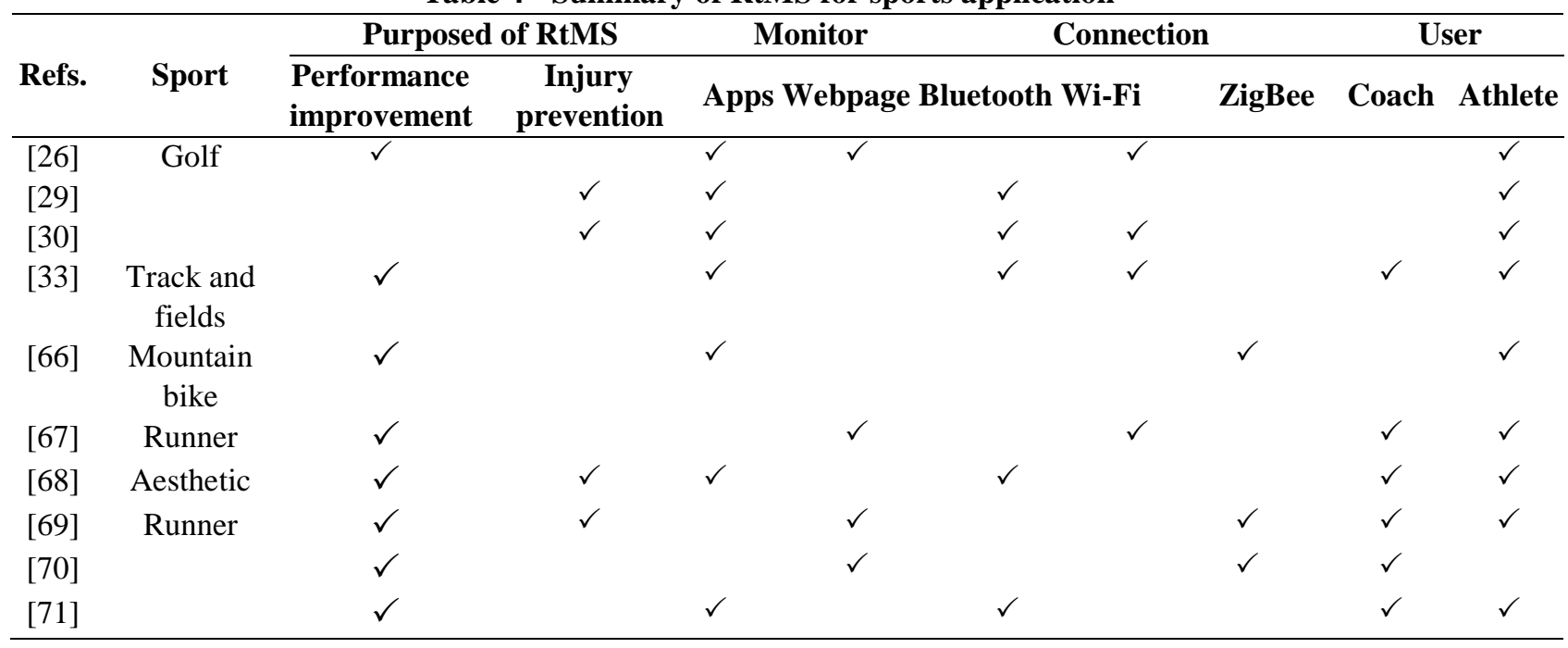

\section{Limitation of Existing Work and Research Opportunities}

In the overall overview of the related works of high jump sport [3], [5], [14]-[17], [6]-[13], at Stage I, the jumper is required to run at moderate speed to gain control of take-off at

Stage II. Thus, the speed is important to control the leg at the ankle before the take-off. Meanwhile, Stage II refers to the take-off that is specifically the period of time for the jumper to be ready to jump. Hence, the ground reaction force at the ankle during that period is very high to gain maximum height of the jump. This is where the highest jump injury takes place. Since before the jump, most of the jumpers used to do heavy jump training to maximise the ground force reaction, this will cause the jumper to overuse the ankle of the leg. Therefore, by running at moderate speed, jumpers can control the take-off and gain better height of jump.

Through these high jump sport parameters conclusion, the design of the wearable device needs to measure the speed of the jumper during the run and the force exerted at the ankle. As the result of the study, the best sensor to track the parameter of speed is the GPS sensor. Meanwhile, FSR sensor will measure force parameter. The high jump sport is done at the track and field stadiums. Thus, wireless connectivity such as Wi-Fi is readily available, by using this wireless connection, it is unnecessary to use Bluetooth to synchronise the data to smartphone, because it will directly send the real-time data to the server or cloud storage via Internet connections and lower the power consumption of smartphones by turning off the Bluetooth connection.

Thus, the idea to make wearable devices with a cheaper price is to combine gateway devices of Wi-Fi integrated MCUs like ESP8266 or Arduino Yun Mini and sensor to become a sensor node. This sensor node will then connect to the server via Internet protocol (IP) address via Internet router as shown in Fig. 7. On the other hand, the Internet router acts as parallel connection of input sensor node, whereas the computer server processes the rule of the item (sensor node) depending on the users' needs and display it to RtMS devices with the help of mobile apps. Through all these processes from Section 2 to 4 , it can be concluded that:

(i) speed and force parameters are important to prevent injuries and improve the athlete's performance;

(ii) high throughput and low latency of the gateway devices are needed to ensure the transmit and receive data are the same;

(iii) low-cost sensors and gateway devices will be combined to become wireless sensor node;

(iv) $\mathrm{Wi}-\mathrm{Fi}$ is the best wireless connection method to connect the sensor nodes to the server to gain real-time communication; and

(v) real-time data is able to be visualised via mobile apps and webpage. 

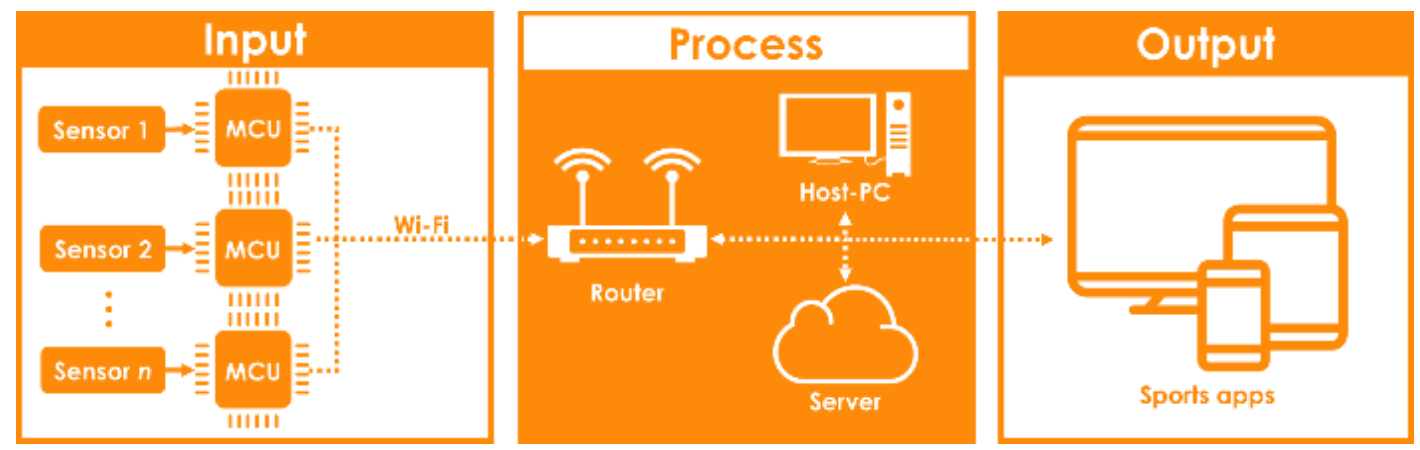

Fig. 7: The overview idea of RtMS input, process and output.

\section{Conclusion}

In this paper, an overview of the activity recognition for high jump wearable device using biosensors, with focus on application from sports are executed. This paper identified previous work of the high jump sport that affect the jumper such as injuries and performances, follows by the analysis of the types and potential issues in sensor related work according to the biosensor technology used in sports application. Last but not least, the best method of processing method used were summarised in terms of gateway device, connection and monitoring system.

As a general reflection, in almost all wearable cases results reported in the related work was achieved by first gathering the main input sensory parameters of the body and then the processing method from the sensor data to the main controller using any wireless connection. As a final point, the process data will be monitored by the display using the smartphone.

\section{Acknowledgment}

The authors would like to thank Zarina Tukiran (UTHM) for sharing her pearls of wisdom with us during the course of this research, and thanks to anonymous reviewers for their so-called insights. We are also immensely grateful to $\mathrm{Dr}$ Mohamad Hairol Jabbar and Dr Chessda Uttraphan Eh Kan for their comments on an earlier version of the manuscript, although any errors are our own and should not tarnish the reputations of these esteemed persons.

\section{References}

[1] K. Lightman, "Silicon gets sporty," IEEE Spectr., vol. 53, no. 3, pp. 48-53, Mar. 2016.

[2] P. Cooke, "High jump analysis," Undergrad. J. Math. Model. One + Two, vol. 5, no. 1, pp. 1-7, 2013.

[3] J. C. C. C. Tan and M. R. Yeadon, "Why do high jumpers use a curved approach?," J. Sports Sci., vol. 23, no. 8, pp. $775-80,2005$.

[4] J. Schiffer, "No. 95: The High Jump," New Stud. Athl., vol. 27, no. 3, pp. 83-99, 2012.

[5] M. A. King, C. Wilson, and M. R. Yeadon, "Evaluation of a torque-driven model of jumping for height," J. Appl. Biomech., vol. 22, no. 4, pp. 264-274, 2006.

[6] J. Isolehto, M. Virmavirta, H. Kyrolainen, and P. Komi, "Biomechanical analysis of the high jump at the 2005 IAAF World Championships in Athletics," New Stud. Athl., vol. 22, no. 2, pp. 17-27, 2007.

[7] J.-P. Goldmann, B. Braunstein, K. Heinrich, M. Sanno, B. Stäudle, W. Ritzdorf, G.-P. Brüggemann, and K. Albracht, "Joint work of the take-off leg during elite high jump," ISBS - Conf. Proc. Arch., vol. 33, no. 1, p. 3, 2016.

[8] B. Dragos, N. Mircea, B. Alexandru, and M. Ilie, "Testing kinematic and ergometer parameters for performing the phase contact-beat - Impulse to the high jump," Ovidius Univ. Ann., vol. 16, no. 2, p. 7, 2016.

[9] A. Burnett, "Jumping Injuries: Their Cause, Possible Prevention and Rehabilitation," elitetrack.com. [Online]. Available: http://elitetrack.com/article_files/jumping-injuries.pdf. [Accessed: 23-Dec-2015].

[10] R. M. Alexander, "Optimum take-off techniques for high and long jumps," Philos. Trans. Biol. Sci., vol. 329, no. 1252, pp. 3-10, 1990.

[11] I. Blažević, L. Antekolović, and M. Mejovšek, "Variability of high jump kinematic parameters in longitudinal follow-up," Kinesiology, vol. 38, no. 1, pp. 63-71, Jun. 2006.

[12] B. Van Gheluwe, P. Roosen, and K. Desloovere, "Rearfoot kinematics during initial take-off of elite high jumpers: estimation of spatial position and orientation of subtalar axis," J. Appl. Biomech., vol. 19, pp. 13-27, 2003.

[13] F. Langer and A. Langerova, "Evaluation of the condition of high jumpers" locomotor system in the prevention of health problems," Kinesiology, vol. 40, no. 1, pp. 107-113, 2008.

[14] C. Wilson, M. M. R. Yeadon, and M. A. King, "Considerations that affect optimised simulation in a running jump for height," J. Biomech., vol. 40, no. 14, pp. 3155-3161, Jan. 2007. 
[15] C. Wilson, M. A. King, and M. Yeadon, "The effects of initial conditions and takeoff technique on running jumps for height and distance," J. Biomech., vol. 44, no. 12, pp. 2207-2212, Aug. 2011.

[16] M. P. Grieg and M. R. Yeadon, "The influence of touchdown parameters on the performance of a high jumper," $J$. Appl. Biomech., vol. 16, no. 4, pp. 367-378, 2000.

[17] M. Čoh, "Biomechanical characteristics of take off action in high jump - A case study," Serbian J. Sport. Sci., vol. 4, no. 4, pp. 127-135, 2010.

[18] D. A. James, L. Fanella, and R. Cusani, "Near Real Time Network Simulation for Team Sports Monitoring," Procedia Eng., vol. 60, pp. 422-427, 2013.

[19] G. Liu, C. Ho, N. Slappey, Z. Zhou, S. E. Snelgrove, M. Brown, A. Grabinski, X. Guo, Y. Chen, K. Miller, J. Edwards, and T. Kaya, "A Wearable Conductivity Sensor for Wireless Real-time Sweat Monitoring," Sensors Actuators B Chem., Dec. 2015.

[20] K. Vuong, "PmSys: a monitoring system for sports athlete load, wellness \& injury monitoring," 2015.

[21] P. Salvo, F. Di Francesco, D. Costanzo, C. Ferrari, M. G. Trivella, and D. De Rossi, “A Wearable Sensor for Measuring Sweat Rate,” IEEE Sens. J., vol. 10, no. 10, pp. 1557-1558, Oct. 2010.

[22] K. E. Iscoe, M. Corcoran, and M. C. Riddell, "High Rates of Nocturnal Hypoglycemia in a Unique Sports Camp for Athletes with Type 1 Diabetes: Lessons Learned from Continuous Glucose Monitoring Systems," Can. J. Diabetes, vol. 32, no. 3, pp. 182-189, Jan. 2008.

[23] A. J. Salazar, A. S. Silva, C. M. Borges, and M. V. Correia, "An initial experience in wearable monitoring sport systems," in Proceedings of the 10th IEEE International Conference on Information Technology and Applications in Biomedicine, 2010, pp. 1-4.

[24] J. Ride, C. Ringuet, D. Rowlands, J. Lee, and D. James, “A Sports Technology Needs Assessment for Performance Monitoring in Swimming," Procedia Eng., vol. 60, pp. 442-447, 2013.

[25] S. Gupta, K. Shivam, D. C. Pandey, and P. Pratap Singh Gaur, "IoT based Patient Health Monitoring system," J. Eng. Technol., vol. 04, no. 3, pp. 2316-2319, 2016.

[26] A. Umek, Y. Zhang, S. Tomažič, and A. Kos, "Suitability of Strain Gage Sensors for Integration into Smart Sport Equipment: A Golf Club Example,” Sensors, vol. 17, no. 4, p. 916, 2017.

[27] M. W. McCarthy, D. A. James, and D. D. Rowlands, "Smartphones: Feasibility for Real-time Sports Monitoring," Procedia Eng., vol. 60, pp. 409-414, 2013.

[28] E. Valchinov, A. Antoniou, K. Rotas, and N. Pallikarakis, "Wireless Health Monitoring System," Wireless Mobile Communication and Healthcare (Mobihealth), 2014 EAI 4th International Conference on. pp. 63-66, 2014.

[29] J. Heaffey, E. Koutsos, and P. Georgiou, "Live demonstration: Wearable device for remote EMG and muscle fatigue monitoring," in 2015 IEEE Biomedical Circuits and Systems Conference (BioCAS), 2015, pp. 1-5.

[30] M. Chen, Y. Ma, and E. Song, "Enabling comfortable sports therapy for patient: A novel lightweight durable and portable ECG monitoring system," in 2013 IEEE 15th International Conference on e-Health Networking, Applications and Services (Healthcom 2013), 2013, pp. 271-273.

[31] D. Morris, B. Schazmann, Y. Wu, S. Coyle, S. Brady, J. Hayes, C. Slater, C. Fay, K. T. Lau, G. Wallace, and D. Diamond, "Wearable sensors for monitoring sports performance and training," in 2008 5th International Summer School and Symposium on Medical Devices and Biosensors, 2008, pp. 121-124.

[32] Y. H. Henao and F. Fruett, "Development of an electromechanical sensor system to monitor sports activities," in 2009 9th International Conference on Electronic Measurement \& Instruments, 2009, pp. 4-6-4-10.

[33] J. P. V. Azcueta, N. C. Libatique, and G. L. Tangonan, "In situ sports performance analysis system using inertial measurement units, high-FPS video camera, and the Android platform," in 7th IEEE International Conference Humanoid, Nanotechnology, Information Technology Communication and Control, Environment and Management (HNICEM), 2014, pp. 1-6.

[34] M. Magno, L. Benini, L. Gaggero, J. P. La Torre Aro, and E. Popovici, “A versatile biomedical wireless sensor node with novel drysurface sensors and energy efficient power management," in 5th IEEE International Workshop on Advances in Sensors and Interfaces IWASI, 2013, pp. 217-222.

[35] B. M. Nigg and M. R. Yeadon, "Biomechanical aspects of playing surfaces," J. Sports Sci., vol. 5, no. 2, pp. 11745, Jan. 1987.

[36] D. J. Farris, G. A. Lichtwark, N. A. T. Brown, and A. G. Cresswell, "The role of human ankle plantar flexor muscletendon interaction and architecture in maximal vertical jumping examined in vivo," J. Exp. Biol., vol. 219, no. 4, pp. 528-534, 2016.

[37] "Long term periodisation of triple jump training," Mod. Athl. Coach, vol. 19, no. 1, pp. 12-14, 1981.

[38] A. J. Salazar, "Wearable monitoring sports systems," Found. Sci. Technol. grant. Gov. Port., 2010.

[39] E. Edwan, J. Zhang, J. Zhou, and O. Loffeld, "Reduced DCM based attitude estimation using low-cost IMU and magnetometer triad," in 2011 8th Workshop on Positioning, Navigation and Communication, 2011, pp. 1-6.

[40] M. B. H. Flores, C. M. B. Siloy, C. Oppus, and L. Agustin, "User-oriented finger-gesture glove controller with hand movement virtualization using flex sensors and a digital accelerometer," in 2014 International Conference on 
Humanoid, Nanotechnology, Information Technology, Communication and Control, Environment and Management (HNICEM), 2014, pp. 1-4.

[41] A. Masdar, B. S. K. K. Ibrahim, D. Hanafi, M. Mahadi Abdul Jamil, and K. A. A. Rahman, "Knee joint angle measurement system using gyroscope and flex-sensors for rehabilitation," in The 6th 2013 Biomedical Engineering International Conference, 2013, pp. 1-4.

[42] M. Sakr and C. Menon, "Regressing force-myographic signals collected by an armband to estimate torque exerted by the wrist: A preliminary investigation," in 2016 IEEE Canadian Conference on Electrical and Computer Engineering (CCECE), 2016, pp. 1-4.

[43] F. Zabihollahy, B. M. Trindade, Y. Ono, and E. D. Lemaire, "Continuous monitoring of mechanical properties of plantar soft tissue for diabetic patients using wearable ultrasonic and force sensors," in 2016 IEEE EMBS International Student Conference (ISC), 2016, pp. 1-4.

[44] B. Zhou, M. Sundholm, J. Cheng, H. Cruz, and P. Lukowicz, "Never skip leg day: A novel wearable approach to monitoring gym leg exercises," in 2016 IEEE International Conference on Pervasive Computing and Communications (PerCom), 2016, pp. 1-9.

[45] M. Wcislik, M. Pozoga, and P. Smerdzynski, "Wireless health monitoring system," IFAC-PapersOnLine, vol. 48, no. 4, pp. 312-317, 2015.

[46] K. W. Nixon, Xiang Chen, and Yiran Chen, "Footfall - GPS polling scheduler for power saving on wearable devices," in 201621 st Asia and South Pacific Design Automation Conference (ASP-DAC), 2016, pp. 563-568.

[47] M. Maheedhar, A. Gaurav, V. Jilla, V. N. Tiwari, and R. Narayanan, "StayFit: A wearable application for Gym based power training," in 2016 38th Annual International Conference of the IEEE Engineering in Medicine and Biology Society (EMBC), 2016, pp. 6290-6293.

[48] S. Thombre, "Performance analysis of IP based WSNs in real time systems," 2016.

[49] A. Bhatt and J. Patoliya, "Cost effective digitization of home appliances for home automation with low-power WiFi devices," in 2016 2nd International Conference on Advances in Electrical, Electronics, Information, Communication and Bio-Informatics (AEEICB), 2016, pp. 643-648.

[50] S. Thombre, R. U1 Islam, K. Andersson, and M. S. Hossain, "Performance analysis of an IP based protocol stack for WSNs," in 2016 IEEE Conference on Computer Communications Workshops (INFOCOM WKSHPS), 2016, pp. 360-365.

[51] M. Gentili, R. Sannino, and M. Petracca, "BlueVoice: Voice communications over Bluetooth Low Energy in the Internet of Things scenario," Comput. Commun., vol. 89, pp. 51-59, 2016.

[52] E. Leão, C. Montez, R. Moraes, P. Portugal, and F. Vasques, "Superframe Duration Allocation Schemes to Improve the Throughput of Cluster-Tree Wireless Sensor Networks," Sensors, vol. 17, no. 2, p. 249, Jan. 2017.

[53] Y. Cheng, S. Xiao, J. Liu, F. Guo, R. Qin, B. Li, and X. Yuan, “An approximate bandwidth allocation algorithm for tradeoff between fairness and throughput in WSN," Wirel. Networks, pp. 1-13, Feb. 2017.

[54] M. M. Kamal, S. S. Moni, and M. S. Alam, "MX-MAC: A Multichannel based low latency asynchronous MAC protocol for Wireless Sensor Networks," in 2016 9th International Conference on Electrical and Computer Engineering (ICECE), 2016, pp. 439-442.

[55] S. Khaliq and S. Henna, "HE-PRMAC: Hop extended pipelined routing enhanced MAC protocol for wireless sensor networks," in 2016 Sixth International Conference on Innovative Computing Technology (INTECH), 2016, pp. 392397.

[56] I. Minakov, R. Passerone, A. Rizzardi, and S. Sicari, "Routing behavior across WSN simulators: The AODV case study," in 2016 IEEE World Conference on Factory Communication Systems (WFCS), 2016, pp. 1-8.

[57] F. A. Aoudia, M. Magno, M. Gautier, O. Berder, and L. Benini, "Poster Abstract: Wake-Up Receivers for Energy Efficient and Low Latency Communication," in 2016 15th ACM/IEEE International Conference on Information Processing in Sensor Networks (IPSN), 2016, pp. 1-2.

[58] "ESP8266EX Overview | Espressif Systems." [Online]. Available: https://espressif.com/en/products/hardware/esp8266ex/overview. [Accessed: 17-Mar-2017].

[59] "Arduino - Arduino Board Yun Mini." [Online]. Available: https://www.arduino.cc/en/Main/ArduinoBoardYunMini. [Accessed: 17-Mar-2017].

[60] P.-W. Lo Frank and M. Q.-H. Meng, "A low cost Bluetooth powered wearable digital stethoscope for cardiac murmur," in 2016 IEEE International Conference on Information and Automation (ICIA), 2016, pp. 1179-1182.

[61] C. Papatsimpa and J. P. M. G. Linnartz, "Coexistence performance model for a large ZigBee lighting sensor network in the $2.4 \mathrm{GHz}$ ISM band," in 2017 IEEE 14th International Conference on Networking, Sensing and Control (ICNSC), 2017, pp. 465-470.

[62] Yun-Liang He, Shu-Qin Geng, Xiao-Hong Peng, Li-Gang Hou, Xiang-Kai Gao, and Jin-Hui Wang, "Design of outdoor air quality monitoring system based on ZigBee wireless sensor network," in IEEE International Conference on Solid-State and Integrated Circuit Technology (ICSICT), 2016, p. 3.

[63] P. Karunakar and A. Chitneni, "A comparative study of wireless protocols: Bluetooth, UWB, ZigBee, and Wi-Fi," in Advance in Electronic and Electric Engineering, 2014, pp. 655-662. 
[64] C. Chilipirea, A. Ursache, D. O. Popa, and F. Pop, "Energy efficiency and robustness for IoT: Building a smart home security system," in 2016 IEEE 12th International Conference on Intelligent Computer Communication and Processing (ICCP), 2016, pp. 43-48.

[65] A. Baniukevic, C. S. Jensen, and H. Lu, "Hybrid Indoor Positioning with Wi-Fi and Bluetooth: Architecture and Performance," in 2013 IEEE 14th International Conference on Mobile Data Management, 2013, pp. $207-216$.

[66] T. Ribeiro, O. Postolache, and P. Passos, "Performance assessment for mountain bike based on WSN and cloud technologies," in 2016 International Conference and Exposition on Electrical and Power Engineering (EPE), 2016, pp. 380-386.

[67] D. D. Rowlands, L. Laakso, T. McNab, and D. A. James, "Cloud based activity monitoring system for health and sport," in The 2012 International Joint Conference on Neural Networks (IJCNN), 2012, pp. 1-5.

[68] E. M. Kidman, M. J. A. D’Souza, and S. P. N. Singh, “A wearable device with inertial motion tracking and vibrotactile feedback for aesthetic sport athletes Diving Coach Monitor," in 2016 10th International Conference on Signal Processing and Communication Systems (ICSPCS), 2016, pp. 1-6.

[69] L. Razoumov, D. J. Bowen, and R. R. Miller, "System and method for wireless monitoring of sports activities," 26 Nov-2013.

[70] N. S. A. Zulkifli, F. K. Che Harun, and N. S. Azahar, "XBee wireless sensor networks for Heart Rate Monitoring in sport training," in 2012 International Conference on Biomedical Engineering (ICoBE), 2012, pp. 441-444.

[71] A. Depari, A. Flammini, S. Rinaldi, and A. Vezzoli, "Multi-sensor system with Bluetooth connectivity for noninvasive measurements of human body physical parameters," Sensors Actuators A Phys., vol. 202, pp. 147-154, Nov. 2013. 